\title{
Q\&A: What can microfluidics do for stem-cell research?
}

\author{
Marie Csete*
}

\section{What can microfluidics do for stem-cell research?}

Stem-cell biology and microfluidics have both been hotbeds of research activity for the past few years, yet neither field has been able to successfully commercialize a clinical 'killer application'. Stem-cell behavior is exquisitely sensitive to environmental cues, and the important cues are difficult to establish, manipulate and quantify in traditional cell culture. Because the microenvironment can be controlled in microfluidics platforms, microfluidics has a lot to offer stem-cell biology and there are many good reasons for the fields to join forces.

\section{What exactly is microfluidics?}

Microfluidics is the characterization and manipulation of fluids on the nanoliter or picoliter scale. The behavior and properties of fluids change as amounts decrease from the macroscale (volumes used for everyday applications) to the microscale. This means that microfluidic devices cannot be built by simply scaling down macroscale devices. For instance, at low microliter volumes, fluids act more like solids, and two fluids flowing alongside each other in a microchannel will not mix well (except by diffusion); therefore, a variety of techniques (pumps, valves, electrokinetics) are used in microfluidics platforms to actuate mixing and fluid flows. Most microfluidics applications in research labs concentrate on the 10 to $100 \mu \mathrm{m}$ scale, basically the diameter of a single cell.

Microfluidics lab-on-a-chip devices allow standard laboratory analyses, such as sample purification, labeling, detection and separation, to be carried out automatically as the sample is moved, via microchannels, to different regions of a chip. Various methods have been used to produce microfluidic devices, but inkjet printers offer an easily accessible way of printing channels and other features directly onto the device. This technique has been used to print precise patterns of proteins or protein gradients onto a surface on which cells can subsequently be cultured to investigate or control their behavior. A technically more

*Correspondence: mariecsete1@gmail.com advanced use of microfluidics is the integration of microchannels with nanoelectrospray emitters for preparing material for mass spectrometry in high-throughput proteomics analyses of biologic samples [1].

\section{What background do you need for microfluidics?}

Physics (in particular fluid dynamics), mechanical engineering, or bioengineering backgrounds, the common feature of these being a strong mathematical foundation.

\section{Why should stem-cell biologists care about miniaturization of cell culture and analysis tools?}

On the one hand, scientists working on the development of pluripotent stem cells for clinical use are encountering a major challenge in scaling up cell cultures for master banks to be used as sources of cell therapies for large numbers of patients. Microfluidics is clearly not the answer to this problem. But on the front end of developing therapies from stem cells, rigorous identification of the starting stem cell and its progeny is a major technical challenge and a regulatory requirement, analogous to the precise chemical identity of a drug. Classically, identification of stem cells is done clonally (at the single-cell level), and it is generally difficult to follow or analyze single cells in mass cell culture. Microfluidics techniques can be used for sensitive discrimination of gene expression (and protein) levels at the single-cell level and they are therefore increasingly useful in stem-cell biology to understand the heterogeneity of stem-cell populations.

Separation of rare stem cells (or rare cancer cell types) from a mixed population is also not easy using flow cytometers developed for clinical use; harsh conditions imposed on the cells during standard flow cytometry mean that cell recovery is low. Microfluidics-based, benchtop flow cytometry allows separation of small numbers of stem cells under direct visualization, and is less damaging to cells than traditional cell sorters. For both analysis and separation, microfluidics offers the means of controlling the cells' environment rigorously. Several groups have also reported that stem cells (and stem cells committed to a particular lineage) can be separated from mixed cell populations using their dielectric properties (electric and magnetic energy). 
In what ways are microfluidics culture conditions superior to those of traditional mass cell culture? Stem-cell fate (growth, death, differentiation, migration) is highly dependent on environmental cues, but the usual cell culture environment does not mimic the in vivo microenvironment in several fundamental ways $(20 \%$ oxygen is unphysiologically high; physiologic fluid flow and shear stresses are not present; three-dimensional environments cannot be standardized), and overall the environment in conventional cell culture is not controllable. For example, $\mathrm{pH}$ inevitably drifts in conventional tissue culture, but in well-designed microfluidics devices, the $\mathrm{pH}$ can be held constant by controlling medium inflow and outflow. In other words, engineers can provide steady-state conditions for cells, as well as fast and predictable changes in the environment surrounding the cells. Of particular importance, the best microfluidics devices are supported by mathematical descriptions of the microenvironment, and information from experiments can be fed back into mathematical models to determine optimal design features to promote specific stem-cell behaviors.

Gradient cues, so important in embryonic development, can be constructed quite precisely on microfluidics devices, as noted above. For example, migration of stem cells in response to chemotactic gradients is often studied in mass cultures using repeated studies in Boyden chambers (two chambers separated by a filter through which cells migrate), but molecular gradients established with microfluidics tools yield inherently more detailed and precise information because gradient characteristics such as slope and concentration can be quantified and correlated to migration behavior. Overall, flexibility in the configuration of microchips is a major advantage of microfluidics-based cell-culture systems, and the ease with which fluid flows can be controlled over time and space.

Human embryonic stem cells (hESCs) are particularly sensitive to handling in culture, and automation of hESC growth and differentiation in vitro on microfluidics platforms produces more standardized outcomes. Many investigators believe that the stress of manual handling of hESCs is an important factor in their instability over time, and therefore automated techniques for passaging and expansion may be a method for overcoming the problem of karyotypic instability.

Three-dimensional mass culture systems are especially 'noisy' and difficult to control using conventional tissueculture methods. Embryoid bodies - floating aggregations of undifferentiated cells - are often used as an intermediate stage in differentiation protocols, and are generated from hESCs by passaging the cells onto nonadherent plates. The resulting embryoid bodies are widely heterogeneous in size unless special engineering protocols are used. This size heterogeneity means that diffusion patterns for signaling through the embryoid bodies and cell-cell interactions are also heterogeneous, resulting in lack of control over the differentiation patterns. Printed topographic features of various shapes on microchips or microchannels are a proven method for gaining control over how cells aggregate. The size and development of embryoid bodies can be controlled with microfluidics techniques, providing a more predictable differentiation pattern and organization of the cells into phenotypically distinct layers. In fact, engineers have successfully manipulated parts of embryoid bodies in different ways using microfluidics tools to alter distinct fates for different parts of the cell aggregates.

The 'micro' in microfluidics plus the configurability of channels can be used to look at simultaneous signals to two parts of a single cell, for example the apical versus basal signals that will be encountered by a polarized cell. In traditional mass culture, cells align in random fashion, and although matrix coatings on tissue-culture plastic can be used to line cells up relative to the matrix, it is impossible to present signals to separate subcellular domains. Epithelial cells are the classical polarized cell in which specific receptors are largely confined to either the apical or basal surface, and signals received at these subcellular domains determine cell function. At the very small scale of microfluidics devices, the apical and basal faces of a cell can be exposed to separate chambers whose composition can be defined and manipulated independently, making it possible to determine the hierarchy of stimuli that determine cell behavior.

An obvious advantage of microfluidics is that it provides economy in terms of reagent use, especially for high-throughput assays. Of course, this economy will only be realized if device fabrication is also inexpensive.

\section{What are some of the major limitations of microfluidics-based cell culture systems?}

Not surprisingly, from a biologist's perspective, the materials-cell interface is still a problem. Polydimethylsiloxane (PMDS) is commonly used to make microchips because it is cheap, optically transparent, gas permeable, and can be manipulated outside a clean room. Although many groups have reported using PDMS chips for hESC studies, my experience is that PDMS has to be considerably modified (and coated), because it is very toxic to the cells. Other, more biocompatible surfaces are available, but the ideal material for exquisitely sensitive cells such as hESCs has not been developed. Again from the biologist's perspective, cellular debris can occlude small channels, so that optimal washing methods in some applications need improvement.

Engineers have pointed out that the best mathematical framework for handling models, such as differentiation, that start at small scales but result in large-scale processes 
is still evolving [2]. So along with the constant improvement in hardware and software needed to make inexpensive devices work optimally, the mathematical tools that make microfluidics approaches so valuable also need continuous refinement. Ultimately, the feedback between biologists using the devices and engineers designing them is the essential key for moving microfluidics-based cell culture forward.

A major issue limiting wide application of microfluidics is that the devices still require experts to operate them, and are not yet biology user-friendly.

\section{What major problems in translational stem-cell biology can be addressed using microfluidics tools?}

Here again, microfluidics techniques afford the ability to define the microenvironment surrounding stem cells. The disease environment into which stem cells will be transplanted is certain to alter their behavior, and is not adequately mimicked in most animal models of disease. Microfluidics-controlled environments can be used to test the tolerance of cells to mechanical and shear forces, gases, oxidants and other extracellular cues that characterize the disease environment. Physical, mechanical and biochemical factors can be tested quantitatively at relatively high throughput on the benchtop using microfluidics to help predict behavior of stem cells in vivo.

Overall, microfluidics tools can be used for spatiotemporal control over the stem-cell microenvironment, so that the ideal ex vivo niche for cell survival and differentiation can be defined quantitatively and in high throughput. Control over the culture environment also allows investigators to perturb cell fate to generate desired outcomes, and to define the limits of physical, mechanical and biochemical factors that are tolerated by stem cells at different stages of differentiation.

\section{What have been the important contributions of microfluidics in biology in general?}

George Whitesides points out that one of the best developed applications of microfluidics is in protein crystallographic studies, to screen the conditions that encourage growth and protection of crystals [3]. For cell biologists, the major impact has been in cell separation, single-cell resolution of the dynamics of gene expression, and insights into how mechanical forces applied to individual cells determine their behavior.

\section{Where can I find out more?}

Cai L, Friedman N, Xie XS: Stochastic protein expression in individual cells at the single molecule level. Nature 2006, 440:358-362.

Cimetta E, Figallo E, Cannizzaro C, Elvassore N, Vunjak-Novakovic G: Micro-bioreactor arrays for controlling cellular environments: Design principles for human embryonic stem cell applications. Methods 2009, 47:81-89.

Melin J, Quake SR: Microfluidic large-scale integration: the evolution of design rules for biological automation. Annu Rev Biophys Biomol Struct 2007, 36:213-231.

Stroock AD, Dertlinger SK, Ajdari A, Mezic I, Stone HA, Whitesides GM: Chaotic mixer for microchannels. Science 2002, 295:647-651.

Tung Y-C, Torisawa Y, Futai N, Takayama S: Small volume low mechanical stress cytometry using computer-controlled Braille display microfluidics. Lab Chip 2007, 7:1497-1503.

Published: 11 February 2010

References

1. Kim W, Guo M, Yang P, Wang D: Microfabricated monolithic multinozzle emitters for nanoelectrospray mass spectrometry. Anal Chem 2007, 79:3703-3707.

2. Bennett MR, Hasty J: Microfluidic devices for measuring gene network dynamics in single cells. Nat Rev Genet 2009, 10:628-638.

3. Whitesides GM: The origins and the future of microfluidics. Nature 2006, 442:368-373

doi:10.1186/jbiol220

Cite this article as: Csete M: Q\&A: What can microfluidics do for stem-cell research? Journal of Biology 2010, 9:1. 